\title{
Robust Simple Adaptive Control with Relaxed Passivity and PID control of a Helicopter Benchmark*
}

\author{
D. Peaucelle $e^{\dagger, \dagger \dagger}$, B. Andrievsky $y^{\ddagger, \ddagger \ddagger}$, \\ V. Mahout ${ }^{\dagger, t \dagger}$, A. Fradkov ${ }^{\ddagger, \ddagger \ddagger}$ \\ † CNRS; LAAS; 7, avenue du Colonel Roche, \\ F-31077 Toulouse, France \\ ${ }^{\dagger \dagger}$ Université de Toulouse; UPS, INSA, INP, ISAE ; LAAS ; \\ F-31077 Toulouse, France \\ ${ }^{\ddagger}$ Institute for Problems of Mechanical Engineering of RAS, \\ 61 Bolshoy av. V.O., St Petersburg, 199178, Russia, \\ 就 Saint Petersburg State University of IT, Mechanics and Optics, \\ Saint Petersburg, Russia
}

October 13, 2010

\begin{abstract}
The paper is dedicated to experimenting a simple LMI procedure to design simple adaptive control laws for 'almost stable' systems. The procedure initially conceived for regulation problems around zero equilibrium point is extended to PID like tracking control problem. LMI results gives conditions to guarantee robustness of the simple adaptive control law with respect to parametric uncertainties. Experiments are done on a 3D helicopter benchmark.
\end{abstract}

\section{Introduction}

Adaptive control is a control strategy proposing to make online modifications of the control law to better reject inevitable uncertainties and perturbations. One such adaptive scheme ([IS96, ÅW89]) relies on estimating online these uncertainties and perturbations and with that knowledge to tune the control law. Another adaptation scheme ([Fra74, KBS94]) proposes to directly tune the control gains with the sole output measurements. Often called direct adaptive control it has the advantage of simplicity compared to estimation/gain-scheduling and other more complex schemes. On the other hand it has the disadvantage to require strong assumptions on the system characteristics in order to prove closed-loop stability. One such direct adaptive control scheme is the passification-based strategy (also called simple adaptive control in [KBS94]). The results presented in this paper enter this framework.

The passivity-based adaptive control framework was first restricted to square systems having same number of inputs and outputs ([Fra74]). Moreover, the systems were assumed to have open-loop hyper-minimum phase properties or equivalently in [KBS94] to be 'almost strictly passive' (i.e. being closed-loop passive with some static output feedback gain). The conditions have been mitigated in [BTH06] and relaxed in [Fra03, Fra08] to non square systems at the expense of finding a linear combination of outputs $G y$ that makes the new weighted system 'almost strictly passive'. Further extension is proposed in [PF08] by the introduction of a parallel feedforward gain

* This work is supported by PICS cooperation \#4281, \# 07-01-92166 between CNRS and RFBR (Russian Foundation for Basic Research). It is also supported by Projects RFBR \# 08-01-00775, \# 09-08-00803, by the Program of Basic Research of OEMPPU RAS \#2 "Control and Safety in Energy and Technical Systems", and by Russian Federal Program "Research and Teaching Cadres", Contract \# 02.740.11.5056. 
(also known as a shunt, see [KBS94]). These extensions conclude that a necessary condition for passivity-based adaptive control is to have a static output feedback $u=F_{0} y$ that renders the closed-loop strictly passive with respect to some output $G y+D u$. Unfortunately, finding these $F_{0}, G, D$ matrices appeared in that paper as a hard problem and does not even guarantee the validity of the adaptive control (the $F_{0}, G, D$ matrices have to satisfy additional LMI conditions). Finally, [PDPM11] gives a new result proving that as soon as a system is 'almost stabilizable' (i.e. closed-loop stable for some static output feedback gain $F_{0}$ ) then a direct adaptive control can be built. This last result is in extended in the present paper to the robust case.

Moreover, the paper proposes a special two term formulation of the adaptive control law. The direct adaptive scheme we study involves? as usual in this framework, a feedback control $u(t)=K(t) y(t)$ where the time-varying gain $K(t)$ is adapted according to a differential equation. This differential equation includes classically a gradient type term $-G y y^{T}$ that drives the feedback gain to stabilizing values. We explore a modification of this structure into a two term control $u(t)=K_{y}(t) y(t)+K_{\eta}(t) \eta(t)$ in which the adaptation differential equation does not involve any squared term of the $\eta$ signal. This modification is proved to be of interest for adaptive PID control. It is then applied to the non-linear model of a helicopter benchmark.

The outline of the paper is as follows. First a section is devoted to the theoretical results about the two term adaptive control and its stability as soon as the system is 'almost stable'. Section 3 extends these results to the robust case. Section 4 exposes the helicopter benchmark control problem which is solved in Section 5. Finally some conclusions are driven.

Notations

$\mathrm{R}^{m \times p}$ is the set of real $m$ by $p$ matrices. $A^{T}$ is the transpose of the matrix $A . \operatorname{Tr}(A)$ is the trace of $A . \mathbf{1}$ and $\mathbf{0}$ are respectively the identity and the zero matrices of appropriate dimensions. For symmetric matrices, $A>(\geq) B$ means that $A-B$ is positive (semi) definite. For a non symmetric matrix $\{A\}^{\mathcal{S}}=A+A^{T} .\|A\|_{D}=\sqrt{\operatorname{Tr}\left(A^{T} D A\right)}$ is the weighted Frobenius norm of A with weight $D>\mathbf{0}$.

$\phi_{D, \alpha}\left(\mathrm{R}^{m \times p} \rightarrow \mathrm{R}^{m \times p}\right)$ is a dead-zone function defined by

$$
\phi_{D, \alpha}(K)=\psi_{\alpha}\left(\|K\|_{D}^{2}\right) \cdot K
$$

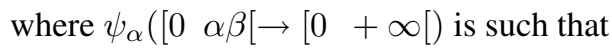

$$
\psi(0 \leq k \leq \alpha)=0, \quad \psi(\alpha \leq k<\alpha \beta)=\frac{k-\alpha}{\alpha \beta-k} .
$$

\section{Main results}

Linear MIMO systems described in state-space are considered

$$
\dot{x}=A x+B u, y=C_{y} x, \eta=C_{\eta} x
$$

where $x \in \mathrm{R}^{n}$ is the state, $u \in \mathrm{R}^{m}$ is the control input, $y \in \mathrm{R}^{p}, \eta \in \mathrm{R}^{q}$ are measured outputs. We denote $C=\left[\begin{array}{cc}C_{y}^{T} & C_{\eta}^{T}\end{array}\right]^{T}$.

It is assumed that the system may be stabilized by a static output feedback

$$
u=F_{0}\left(\begin{array}{c}
y \\
\eta
\end{array}\right)=F_{0 y} y+F_{0 \eta} \eta
$$

and one such gain is assumed to be known. Our goal is to provide design conditions for the simple adaptive control:

$$
u(t)=K_{y}(t) y(t)+K_{\eta}(t) \eta(t)
$$

with the following type of adaptation:

$$
\begin{aligned}
& \dot{K}_{y}(t)=-G_{y}\left(\begin{array}{c}
y(t) \\
\eta(t)
\end{array}\right) y^{T}(t) \Gamma_{y}-\phi_{D_{y}, \alpha_{y}}\left(K_{y}(t)-F_{0 y}\right) \Gamma_{y}, \\
& \dot{K}_{\eta}(t)=-G_{\eta} y(t) \eta^{T}(t) \Gamma_{\eta}-\phi_{D_{\eta}, \alpha_{\eta}}\left(K_{\eta}(t)-F_{0 \eta}\right) \Gamma_{\eta} .
\end{aligned}
$$

No assumption is made about the closed-loop passivity but the following proposition gives a way to define passive outputs for that closed-loop system. 
Proposition 1 If $A\left(F_{0}\right)=A+B F_{0} C$ is asymptotically stable then there exists $\left(P, G_{y}, D_{y}, G_{\eta}, D_{\eta}\right)$ solution to the following LMI problem: $P>\mathbf{0}$,

$$
\left[\begin{array}{ccc}
\left\{P A\left(F_{0}\right)\right\}^{\mathcal{S}} & P B-C^{T} G_{y}^{T} & P B-C_{y}^{T} G_{\eta}^{T} \\
B^{T} P-G_{y} C & -D_{y} & \mathbf{0} \\
B^{T} P-G_{\eta} C_{y} & \mathbf{0} & -D_{\eta}
\end{array}\right]<\mathbf{0} .
$$

The pairs $\left(G_{y}, D_{y}\right)$ and $\left(G_{\eta}, D_{\eta}\right)$ are then such that the two following systems are passive

$$
\begin{aligned}
& \dot{x}=A\left(F_{0}\right) x+B w, \quad z_{y}=G_{y} C x+1 / 2 D_{y} w \\
& \dot{x}=A\left(F_{0}\right) x+B w, z_{\eta}=G_{\eta} y+1 / 2 D_{\eta} w .
\end{aligned}
$$

The proof is quite simple and is a direct extension of the one to be found in [PDPM11].

Having obtained some passivity property of the system, the following theorem allows to prove closed-loop stability with a simple adaptive control.

Theorem 1 Let $\beta>1$ be a given scalar. If $\left(F_{0}, G_{y}, D_{y}, G_{\eta}, D_{\eta}\right)$ are feasible values for LMIs (1), then there exists $\left(Q>\mathbf{0}, R_{y}, R_{\eta}, T_{y}, T_{\eta}, F_{y}, F_{\eta}, \alpha_{y}, \alpha_{\eta}\right)$ solutions to the following LMI problem

$$
\begin{gathered}
{\left[\begin{array}{cc}
R_{y} & Q B-C^{T} G_{y}^{T} \\
B^{T} Q-G_{y} C & D_{y}
\end{array}\right] \geq \mathbf{0},} \\
{\left[\begin{array}{cc}
R_{\eta} & Q B-C_{y}^{T} G_{\eta}^{T} \\
B^{T} Q-G_{\eta} C_{y} & D_{\eta}
\end{array}\right] \geq \mathbf{0},} \\
{\left[\begin{array}{cc}
T_{y} & \left(F_{y}-F_{0 y}\right)^{T} \\
\left(F_{y}-F_{0 y}\right) & D_{y}^{-1}
\end{array}\right] \geq \mathbf{0}, \operatorname{Tr}\left(T_{y}\right) \leq \alpha_{y},} \\
{\left[\begin{array}{cc}
T_{\eta} & \left(F_{\eta}-F_{0 \eta}\right)^{T} \\
\left(F_{\eta}-F_{0 \eta}\right) & D_{\eta}^{-1}
\end{array}\right] \geq \mathbf{0}, \operatorname{Tr}\left(T_{\eta}\right) \leq \alpha_{\eta},} \\
\left\{Q A\left(F_{0}\right)\right\}^{\mathcal{S}} \\
+\alpha_{y} \beta C_{y}^{T} C_{y}+R_{y}+\left\{C^{T} G_{y}^{T}\left(F_{y}-F_{0 y}\right) C_{y}\right\}^{\mathcal{S}} \\
+\alpha_{\eta} \beta C_{\eta}^{T} C_{\eta}+R_{\eta}+\left\{C_{y}^{T} G_{\eta}^{T}\left(F_{\eta}-F_{0 \eta}\right) C_{\eta}\right\}^{\mathcal{S}}<\mathbf{0 .} .
\end{gathered}
$$

The solution is such that the adaptive controller stabilizes the system.

The proof follows the lines of the proof in [PDPM11]. It is not produced here to give sufficiently space for discussing its application on a concrete problem.

At this stage the results can seem confusing. Theorem 1 gives, at the knowledge of a stabilizing SOF gain $F_{0}$, an alternative adaptive controller, more complex that the SOF control. What is expected is that the adaptive law will have better robustness properties than the original SOF and this issue is discussed in the following.

Remark 1 The adaptive control law is such that the gain $K$ varies around the original stabilizing gain $F_{0}$. It cannot escape from the bounded set

$$
\left\|K_{y}(t)-F_{0 y}\right\|_{D_{y}}^{2} \leq \alpha_{y} \beta,\left\|K_{\eta}(t)-F_{0 \eta}\right\|_{D_{\eta}}^{2} \leq \alpha_{\eta} \beta
$$

even in the presence of bounded noise (see [PDPM11] and [PFA08]) and as the system stabilizes it converges to the bounded set

$$
\left\|K_{y}(\infty)-F_{0 y}\right\|_{D_{y}}^{2} \leq \alpha_{y},\left\|K_{\eta}(\infty)-F_{0 \eta}\right\|_{D_{\eta}}^{2} \leq \alpha_{\eta} .
$$

Trivially one cannot expect any improvement from the adaptive control if these sets are small. One should therefore seek for solutions maximizing $\alpha_{y}$ and $\alpha_{\eta}$ (when solving LMIs of Theorem 1) while minimizing $D_{y}$ and $D_{\eta}$ (when solving LMIs of Proposition 1). 


\section{Guaranteed robustness}

As in [PF08] we shall consider constant polytopic uncertainties. Results may as well be extended following the lines of [PKP09] to the case of time-varying uncertainties. The difference is then that convergence of the state $x$ to an equilibrium point may no more be proved but informations on the derivatives of the uncertainties guarantee that the state remains in a compact neighborhood of the equilibrium. Conservative over-bounds on the size of this neighborhood may be computed. They often appear to be very conservative and, in practice, neighborhoods shrink to the equilibrium point. Moreover, if the derivatives of the uncertainties go to zero, the neighborhood will inevitably shrink to the equilibrium.

The uncertain system is assumed to have uncertainties only on the differential equation

$$
\dot{x}=A(\xi) x+B(\xi) u, \quad y=C_{y} x, \quad \eta=C_{\eta} x .
$$

The uncertainties are assumed affine

$$
\left[\begin{array}{ll}
A(\xi) & B(\xi)
\end{array}\right]=\sum_{v=1}^{\bar{v}} \xi_{v}\left[\begin{array}{ll}
A^{[v]} & B^{[v]}
\end{array}\right]
$$

of polytopic type $\xi \in \Xi=\left\{\xi_{v} \geq 0, \quad \sum_{i=1}^{\bar{v}} \xi_{v}=1\right\}$.

Denote $A^{[v]}\left(F_{0}\right)=A^{[v]}+B^{[v]} F_{0} C$. The following proposition gives conditions for robust passivity of the closed-loop with the SOF gain $F_{0}$

Proposition 2 If there exists $\left(P^{[v]}, G_{y}, D_{y}, G_{\eta}, D_{\eta}\right)$ solution for all $v=1 \ldots \bar{v}$ to the following LMIs: $P^{[v]}>\mathbf{0}$,

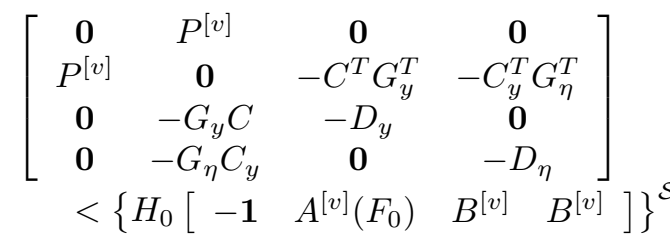

then the pairs $\left(G_{y}, D_{y}\right)$ and $\left(G_{\eta}, D_{\eta}\right)$ are then such that the two following systems are passive for all $\xi \in \Xi$

$$
\begin{array}{ll}
\dot{x}=A\left(\xi, F_{0}\right) x+B(\xi) w, & z_{y}=G_{y} C x+1 / 2 D_{y} w \\
\dot{x}=A\left(\xi, F_{0}\right) x+B(\xi) w, & z_{\eta}=G_{\eta} y+1 / 2 D_{\eta} w .
\end{array}
$$

The following Theorem gives conditions for robust stability with the adaptive control.

Theorem 2 Let $\left(\beta>1, F_{0}, G_{y}, D_{y}, G_{\eta}, D_{\eta}\right)$ be given, if there exists $\left(Q^{[v]}>\mathbf{0}, R^{[v]}, H, H_{R} T_{y}^{[v]}, T_{\eta}^{[v]}, F_{y}^{[v]}\right.$, $\left.F_{\eta}^{[v]}, \alpha_{y}, \alpha_{\eta}\right)$ solutions for all $v=1 \ldots \bar{v}$ to the following LMIs

$$
\begin{aligned}
& {\left[\begin{array}{cccc}
R^{[v]} & -C^{T} G_{y}^{T} & -C_{y}^{T} G_{\eta}^{T} & Q^{[v]} \\
-G_{y} C & D_{y} & \mathbf{0} & \mathbf{0} \\
-G_{\eta} C_{y} & \mathbf{0} & D_{\eta} & \mathbf{0} \\
Q^{[v]} & \mathbf{0} & \mathbf{0} & \mathbf{0}
\end{array}\right]}
\end{aligned}
$$

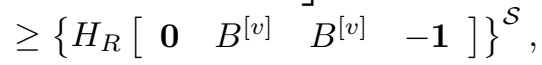

$$
\begin{aligned}
& {\left[\begin{array}{cc}
T_{y}^{[v]} & \left(F_{y}^{[v]}-F_{0 y}\right)^{T} \\
\left(F_{y}^{[v]}-F_{0 y}\right) & D_{y}^{-1}
\end{array}\right] \geq \mathbf{0}, \quad \operatorname{Tr}\left(T_{y}^{[v]}\right) \leq \alpha_{y},} \\
& {\left[\begin{array}{cc}
T_{\eta}^{[v]} & \left(F_{\eta}^{[v]}-F_{0 \eta}\right)^{T} \\
\left(F_{\eta}^{[v]}-F_{0 \eta}\right) & D_{\eta}^{-1}
\end{array}\right] \geq \mathbf{0}, \quad \operatorname{Tr}\left(T_{\eta}^{[v]}\right) \leq \alpha_{\eta},}
\end{aligned}
$$




$$
\begin{gathered}
\left.\quad \begin{array}{cc}
L^{[v]} & Q^{[v]} \\
Q^{[v]} & \mathbf{0}
\end{array}\right]<\left\{H\left[A^{[v]}\left(F_{0}\right)-\mathbf{1}\right]\right\}^{\mathcal{S}} \\
L^{[v]}=R^{[v]}+\alpha_{y} \beta C_{y}^{T} C_{y}+\left\{C^{T} G_{y}^{T}\left(F_{y}^{[v]}-F_{0 y}\right) C_{y}\right\}_{\mathcal{S}}^{\mathcal{S}} \\
\quad+\alpha_{\eta} \beta C_{\eta}^{T} C_{\eta}+\left\{C_{y}^{T} G_{\eta}^{T}\left(F_{\eta}^{[v]}-F_{0 \eta}\right) C_{\eta}\right\}^{\mathcal{S}} .
\end{gathered}
$$

then the adaptive controller stabilizes robustly the system.

Proof Proposition 2 and Theorem 2 are obtained by classical robustness arguments for affine polytopic systems. See for example [PF08].

It should be noted that while Theorem 1 is defined assuming $F_{0}$ is a stabilizing SOF, no such assumption is made in Theorem 2. In Theorem 1 the assumption is used for guaranteeing the existence of a solution, but solutions may exist even if $A\left(F_{0}\right)$ is unstable. For initialization of the data $\left(\beta>1, F_{0}, G_{y}, D_{y}, G_{\eta}, D_{\eta}\right)$ Proposition 1 can used for the nominal system (center of the polytope) and $F_{0}$ needs to stabilize this nominal system but may not be a robust control. No assumption is made in Theorem 2 about the robust stabilization with a unique, parameterindependent controller. Studying the proof provided in [PDPM11] one can nevertheless see that some robustly stabilizing parameter-dependent static output-feedback should exist for the theorem to hold. In the present case this feedback gain is

$$
F(\xi)=\sum_{v=1}^{\bar{v}} \xi_{v}\left[\begin{array}{ll}
F_{y}^{[v]} & F_{\eta}^{[v]}
\end{array}\right]
$$

but cannot be implemented because the parameters are not measured.

\section{The PID case}

Let a linear plant

$$
\dot{\mathrm{x}}=\mathrm{Ax}+\mathrm{B} u+d, \quad \mathrm{y}=\mathrm{Cx}
$$

for which we aim at designing an adaptive PID controller:

$$
u(t)=K_{P}(t)\left(\mathrm{y}(t)-\mathrm{y}^{*}\right)+K_{I}(t) \int_{0}^{t}\left(\mathrm{y}(\tau)-\mathrm{y}^{*}\right) d \tau+K_{D}(t) \hat{\mathrm{y}}(t)
$$

capable of driving the closed-loop system outputs to reference constant values $\mathrm{y}^{*}$ whatever bounded steady-state disturbance $d(t) \stackrel{t \rightarrow \infty}{\longrightarrow} d^{*}$. The derivative of the output is either assumed to be measured $\hat{\mathrm{y}}=\dot{\mathrm{y}}$ or obtained through a linear filter

$$
\dot{x}_{f}=A_{f} x_{f}+B_{f} \mathrm{y}, \hat{\mathrm{y}}=C_{f} x_{f}+D_{f} \mathrm{y} .
$$

The integral component of the control guarantees, if the closed-loop is stable, the convergence of $y$ to $\mathrm{y}^{*}$. To design the parameters of the adaptive PID the problem is converted into the stabilization problem of the following model

$$
\begin{aligned}
& \dot{x}=\left[\begin{array}{ccc}
\mathrm{A} & \mathbf{0} & \mathbf{0} \\
\mathrm{C} & \mathbf{0} & \mathbf{0} \\
B_{f} \mathrm{C} & \mathbf{0} & A_{f}
\end{array}\right] x+\left[\begin{array}{l}
\mathrm{B} \\
\mathbf{0} \\
\mathbf{0}
\end{array}\right] u \\
& y=\left[\begin{array}{ccc}
\mathrm{C} & \mathbf{0} & \mathbf{0} \\
D_{f} \mathrm{C} & \mathbf{0} & C_{f}
\end{array}\right] x, \eta=\left[\begin{array}{lll}
\mathbf{0} & \mathbf{1} & \mathbf{0}
\end{array}\right] x
\end{aligned}
$$

where $x^{T}=\left(\begin{array}{lll}\mathrm{x}^{T} & \int \mathrm{y}^{T} & x_{f}^{T}\end{array}\right), y^{T}=\left(\begin{array}{ll}\mathrm{y}^{T} & \hat{\mathrm{y}}^{T}\end{array}\right)$ and $\eta=\int \mathrm{y}$.

The problem is formalized appropriately to apply results of the previous sections. The important issue here is the choice of the two outputs $y$ and $\eta$. This is done to make possible the convergence of the gains. Given a non-zero reference $\mathrm{y}^{*} \neq 0$, if the system is asymptotically stable, $\mathrm{y}-\mathrm{y}^{*}$ and the 'derivative' $\hat{\mathrm{y}}$ will converge to zero while the integral $\int_{0}^{t}\left(\mathrm{y}(\tau)-\mathrm{y}^{*}\right) d \tau$ will converge to a constant value. No adaptive scheme is therefore possible based on a adaptation law depending of the square of this signal. And indeed with the proposed structure of adaptation there is no such term. The adaptation will stop as soon as the system has converged to the reference. 


\section{THE “HELICOPTER” benchmark}

The 3DOF helicopter considered setup is manufactured by Quanser Consulting Inc., www . quanser.com.This setup was modified under demand of LAAS-CNRS for implementation and testing robust control laws. It consists of a base on which a long arm is mounted. The arm carries the helicopter body on one end and a counterweight on the other end. The arm can tilt on an elevation axis as well as swivel on a vertical (travel) axis. Quadrature optical encoders mounted on these axes measure the elevation and travel of the arm. The helicopter body, which is mounted at the end of the arm, is free to pitch about the pitch axis. The pitch angle is measured via a third encoder. Two motors with propellers mounted on the helicopter body can generate a force proportional to the voltage applied to them. The force, generated by the propellers, causes the helicopter body to lift off the ground and/or to rotate about the pitch axis. All electrical signals to and from the arm are transmitted via a slipping with eight contacts. The system is also equipped with a motorized lead screw that can drive a mass along the main arm in order to impose known controllable disturbances (the so-called Active Disturbance Option, ADO).

Following notation is used through the paper (see Fig. 1): $\theta$ is pitch angle; $\varepsilon$ is elevation angle; $\lambda$ is travel angle; $v_{f}, v_{r}$ is control voltages of the "front" (conditionally) and the "rear" motors. Denote $u=v_{f}-v_{r}$ and $v=v_{f}+v_{r}$, the control voltages $v_{f}$ and $v_{r}$, applied to the front and rear motors are calculated based on the command signals $u, v$ as follows:

$$
v_{f}=0.5(v+u), \quad v_{r}=0.5(v-u) .
$$

The motor control voltages have saturation level $10 \mathrm{~V}$ on the magnitude.

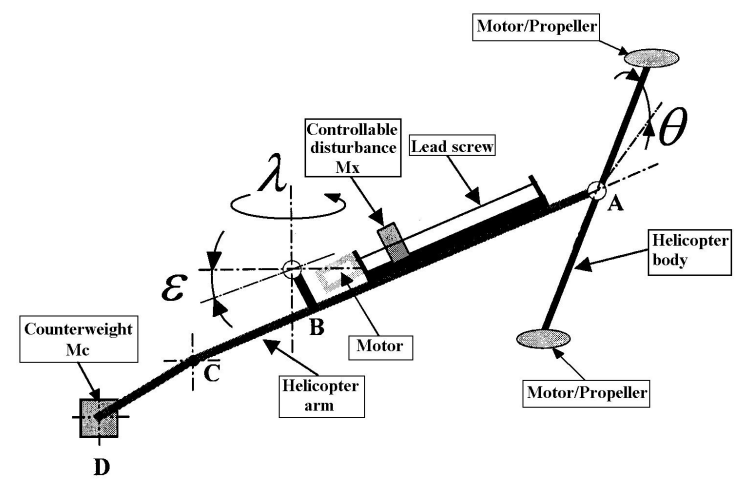

Figure 1: Lay-out of the "Helicopter".

Let us use the following simplifying assumptions to obtain the "Helicopter" model: the "Helicopter" is considered as a rigid body, i.e. it is assumed that the long arm of the "Helicopter" rotates about the long axis together with the crossbar, and bending of structural components is neglected; the gyroscopic torques, developed by motor/propeller pairs are neglected; dependence of motor/propeller force gain on the "Helicopter" airspeed is neglected; influence of aerodynamical pressure forces on the "Helicopter" body is neglected; dry friction in the pivots is neglected. Based on the given assumptions, the following simplified model is obtained (see also [KKGZ06, KKK04, PFA07]):

$$
\left\{\begin{array}{l}
\ddot{\varepsilon}=-a_{1 \varepsilon} \dot{\varepsilon}-a_{0 \varepsilon} \sin \left(\varepsilon-\varepsilon_{0}\right)+b_{\varepsilon} \cos \theta \cdot v \\
\ddot{\theta}=-a_{1 \theta} \dot{\theta}-a_{0 \theta} \sin \left(\theta-\theta_{0}\right)+b_{\theta} \cos \varepsilon \cdot u \\
\ddot{\lambda}=-a_{1 \lambda} \dot{\lambda}+b_{\lambda} \sin \theta \cdot v
\end{array}\right.
$$

where $a_{\star}, b_{\star}, \theta_{0}$ and $\epsilon_{0}$ are uncertain plant model parameters for which identification experiments allow to give upper and lower bounds (see [PFA07]). The constants $\theta_{0}$ and $\varepsilon_{0}$ denote pitch and elevation balance angles. The value of $\varepsilon_{0}$ depends on the weight $M_{x}$ position (see Fig. 1) and may be varying in different experiments at the operators commands (the "ADO option"). 
We assume in the following that only positive values of the normal force command signal $v(t)$ are applied, $v \in[0.5,10]$. This means that we are interested only in positive values of the desired elevation angle. Twodirectional travel motion in this case may be achieved by changing the sign of the pitch angle (due to multiplying $v(t)$ by $\sin \theta$ in the third equation of (16)). The elevation angle has physical limitations $\epsilon \in[-520]$ deg. Moreover the pitch angle has mechanical restrictions as $|\theta| \leq 90 \mathrm{deg}$ but in the following we assume that $\theta(t)$ is kept within the working area $|\theta| \leq 60 \mathrm{deg}$.

With all these assumptions and uncertainties, the system also writes as the following time-varying linear system

$$
\left\{\begin{array}{l}
\ddot{\varepsilon}=-\mathrm{a}_{1 \varepsilon} \dot{\varepsilon}(t)-\mathrm{a}_{0 \varepsilon}(t) \varepsilon(t)+\mathrm{b}_{\varepsilon}(t) v(t)+d_{\varepsilon}(t) \\
\ddot{\theta}=-\mathrm{a}_{1 \theta} \dot{\theta}-\mathrm{a}_{0 \theta}(t) \theta(t)+\mathrm{b}_{\theta}(t) u(t)+d_{\theta}(t) \\
\ddot{\lambda}=-\mathrm{a}_{1 \lambda} \dot{\lambda}+\mathrm{b}_{\lambda}(t) \theta(t)
\end{array}\right.
$$

where the time-varying parameters are defined as:

$$
\begin{array}{ll}
\mathrm{a}_{1 \epsilon}=a_{1 \epsilon} & \in\left[\begin{array}{ll}
0.02 & 0.15
\end{array}\right] \\
\mathrm{a}_{0 \varepsilon}(t)=a_{0 \varepsilon} \cos \varepsilon_{0} \sin \varepsilon(t) / \varepsilon(t) & \in\left[\begin{array}{ll}
3 & 5
\end{array}\right] \\
\mathrm{b}_{\varepsilon}(t)=b_{\varepsilon} \cos \theta(t) & \in\left[\begin{array}{ll}
0.2 & 0.4
\end{array}\right] \\
d_{\varepsilon}(t)=a_{0 \varepsilon} \sin \varepsilon_{0} \cos \varepsilon(t) & \in\left[\begin{array}{ll}
-1.3 & 0
\end{array}\right] \\
\mathrm{a}_{1 \theta}=a_{1 \theta} & \in\left[\begin{array}{ll}
0.02 & 0.15
\end{array}\right] \\
\mathrm{a}_{0 \theta}(t)=a_{0 \theta} \cos \theta_{0} \sin \theta(t) / \theta(t) & \in\left[\begin{array}{ll}
0.3 & 0.7
\end{array}\right] \\
\mathrm{b}_{\theta}(t)=b_{\theta} \cos \varepsilon(t) & \in\left[\begin{array}{ll}
0.2 & 0.4
\end{array}\right] \\
d_{\theta}(t)=a_{0 \theta} \sin \theta_{0} \cos \theta(t) & \in\left[\begin{array}{ll}
-0.14 & 0.14
\end{array}\right] \\
\mathrm{a}_{1 \lambda}=a_{1 \lambda} & \in\left[\begin{array}{ll}
0.02 & 0.05
\end{array}\right] \\
\mathrm{b}_{\lambda}(t)=b_{\lambda} v(t) \sin \theta(t) / \theta(t) & \in\left[\begin{array}{ll}
0.05 & 0.1
\end{array}\right] .
\end{array}
$$

Written in this way, the model exhibits a decoupling of the elevation dynamics actuated by $v$ on one side and the pitch-travel dynamics actuated by $u$ on the other. A decoupled PID-type control is therefore considered:

$$
\begin{aligned}
& v=K_{P \varepsilon}\left(\epsilon-\epsilon^{*}\right)+K_{I \varepsilon} \int\left(\varepsilon-\varepsilon^{*}\right) d \tau+K_{D \varepsilon} \dot{\varepsilon} \\
& u=K_{P \lambda}\left(\lambda-\lambda^{*}\right)+K_{I \lambda} \int\left(\lambda-\lambda^{*}\right) d \lambda+K_{D \lambda} \dot{\lambda} \\
& +K_{P \theta} \theta+K_{D \theta} \dot{\theta}
\end{aligned}
$$

\section{Static and adaptive control of the "helicopter"}

To apply the results of the previous sections it is first needed to conceive stabilizing static feedback gains:

$$
\begin{aligned}
& F_{0 v}=\left[\begin{array}{llll}
F_{0 P \varepsilon} & F_{0 I \varepsilon} & F_{0 D \varepsilon}
\end{array}\right] \\
& F_{0 u}=\left[\begin{array}{lllll}
F_{0 P \lambda} & F_{0 I \lambda} & F_{0 D \lambda} & F_{0 P \theta} & F_{0 D \theta}
\end{array}\right]
\end{aligned}
$$

for both of the PID laws. In this "helicopter" example, both positions $\epsilon, \lambda, \theta$ and rotation speeds $\dot{\epsilon}, \dot{\lambda}, \dot{\theta}$ are measured. Thus, the static feedbacks can be written as state-feedback controls on the two augmented systems of the type (14):

$$
\begin{gathered}
\dot{x}_{\varepsilon}=\left[\begin{array}{ccc}
0 & 0 & 1 \\
1 & 0 & 0 \\
-\mathrm{a}_{0 \varepsilon} & 0 & -\mathrm{a}_{1 \varepsilon}
\end{array}\right] x_{\varepsilon}+\left[\begin{array}{c}
0 \\
0 \\
\mathrm{~b}_{\varepsilon}
\end{array}\right] v \\
\dot{x}_{\lambda}=\left[\begin{array}{cccccc}
0 & 0 & 1 & 0 & 0 \\
1 & 0 & 0 & 0 & 0 \\
0 & 0 & -\mathrm{a}_{1 \lambda} & \mathrm{b}_{\lambda} & 0 \\
0 & 0 & 0 & 0 & 1 \\
0 & 0 & 0 & -\mathrm{a}_{0 \theta} & -\mathrm{a}_{1 \theta}
\end{array}\right] x_{\lambda}+\left[\begin{array}{c}
0 \\
0 \\
0 \\
0 \\
\mathrm{~b}_{\theta}
\end{array}\right] u
\end{gathered}
$$

with $x_{\varepsilon}=\left(\begin{array}{llll}\epsilon & \int \varepsilon & \dot{\varepsilon}\end{array}\right)^{T}$ and $x_{\lambda}=\left(\begin{array}{llllll}\lambda & \int \lambda & \dot{\lambda} & \theta & \dot{\theta}\end{array}\right)^{T}$. 
Such state-feedback gains may be designed using, for example, the RoMulOC toolbox ([Pea05],[PA06]). For space limitations reasons we do not detail this static feedback design step. For both gains specifications were in terms of pole location plus, in the travel-pitch case, a specification to minimize the pitch peak in response to steps on the travel reference. The obtained gains are

$$
\begin{aligned}
& F_{0 v}=-\left[\begin{array}{lrl}
44.089 & 45.750 & 26.518
\end{array}\right] \\
& F_{0 u}=-\left[\begin{array}{lrlll}
174.834 & 9.846 & 1026.224 & 172.897 & 47.646
\end{array}\right] .
\end{aligned}
$$

Solving inequalities of Proposition 2 for the elevation model using $F_{0 v}$ as a stabilizing gain one finds

$$
\begin{gathered}
G_{y v}=\left[\begin{array}{lll}
6.210 & 4.890 & 0.098
\end{array}\right], \quad D_{y v}=0.0010 \\
G_{\eta v}=\left[\begin{array}{ll}
6.328 & 4.671
\end{array}\right], D_{\eta v}=0.0011 .
\end{gathered}
$$

Solving the inequalities this time for the travel-pitch models using $F_{0 u}$ as a stabilizing gain gives

$$
\begin{gathered}
G_{y u}=\left[\begin{array}{lllll}
6.642 & 45.429 & 1.460 & 4.304 & 0.003
\end{array}\right], \\
G_{\eta u}=\left[\begin{array}{llll}
6.665 & 45.096 & 1.449 & 4.302
\end{array}\right] \\
D_{y u}=3.245 \cdot 10^{-4}, \quad D_{\eta u}=3.257 \cdot 10^{-4}
\end{gathered}
$$

In both cases the LMIs were coded using YALMIP of [Löf04] and with SeDuMi solver of [Stu99]. Computation time for the elevation model (polytope with 8 vertices, system of order 3) is 1.9 seconds on Mac OSX computer with $2.66 \mathrm{GHz}$ Inter Core 2 Duo processor. For the travel-pitch model (polytope with 32 vertices, system of order $5)$ the computation time was 4.3 seconds. The proposed solutions are not any feasible solutions but those that minimize $D_{y}+D_{\eta}$. In this manner one can expect to get larger adaptation regions (see Remark 1).

The final step is to compute the scalars $\alpha_{v}$ and $\alpha_{u}$ that define the domains in which the adaptation gains evolve while preserving stability. These scalars are computed using Theorem 2 . The obtained values are

$$
\begin{gathered}
\alpha_{y v}=1012.6, \quad \alpha_{\eta v}=0.343, \\
\alpha_{y u}=86.803, \quad \alpha_{\eta u}=8.210 \cdot 10^{-4}
\end{gathered}
$$

If implemented as is, the gains $K_{y v}$ are allowed to vary too far away from the stabilizing value $F_{0 v}$. In practice the adaptation then drives the gains to values reducing the time response but this is done at the expense of larger values of the control input $u$. In practice this input is saturated (limited capacity of actuators) and the adaptation thus drives the gains to destabilizing situations. To avoid this, we chose to limit the adaptation and took $\alpha_{y v}=0.1$. The gains $K_{y v}$ are therefore kept close to the static ones.

Simulations are done on the non-linear model with the following choice of parameters

$$
\begin{array}{llll}
a_{1 \epsilon}=0.085, & a_{0 \varepsilon}=4.5, & b_{\varepsilon}=0.3, & \varepsilon_{0}=0, \\
a_{1 \theta}=0.085, & a_{0 \theta}=0.5, & b_{\theta}=0.3, & \theta_{0}=0, \\
a_{1 \lambda}=0.035, & & b_{\lambda}=0.075 . &
\end{array}
$$

Two square repeating sequences are applied as references on the elevation and the travel. The elevation reference switches between $1^{\circ}$ and $10^{\circ}$ every 10 seconds. The travel reference switches between 0 and $9^{\circ}$ every 70 seconds.

Figure 2, 3 and 4 show the time histories of the travel, pitch and elevation angles respectively. The dotted lines are the square reference signals. The dashed lines are the responses with static feedback gains $F_{0 u}, F_{0 v}$. The solid lines are the responses with adaptive PID control. The elevation plot being hardly readable Figure 5 gives a zoom of this plot.

Some explanations about the simulations: Firstly notice the answer of the travel axis is not identical in all situations. it very much depends on wether the helicopter is at high of low elevations. In the former case (first travel rise) the force of the propellers $(v)$ is large at the instant of the step, it generates a strong torque for travel and a rapid response. In the latter case (second travel rise) the force of the propellers is small and has less effect. Secondly notice in Figure 5 disturbance at time 265. This disturbance is due to the coupling between the axes. At that instant the step on the travel angle generates a strong pitch angle $\theta$. The vertical component of the propellers force is $\cos \theta \cdot v$, it suddenly decreases thus creating this dropping of the helicopter. These two behaviors of the non-linear model are coherent with what is observed on the real plant. 


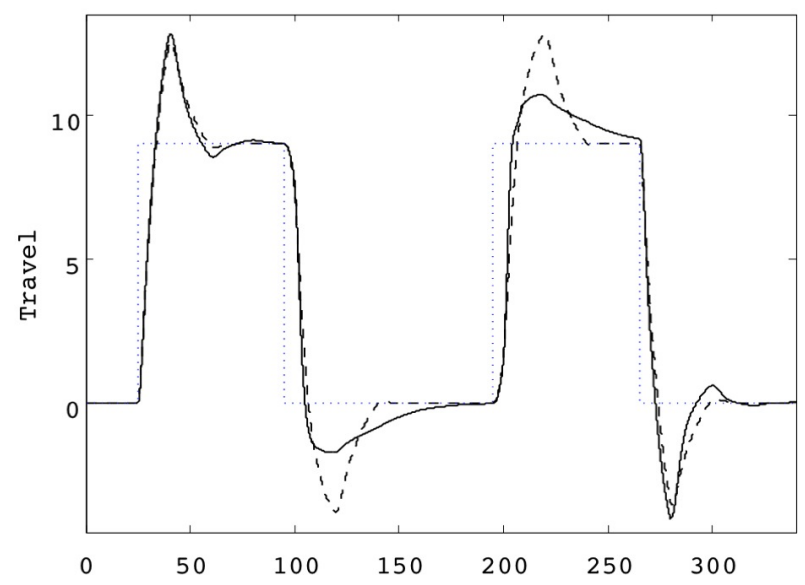

Figure 2: Times histories of travel angle $\lambda(t)$

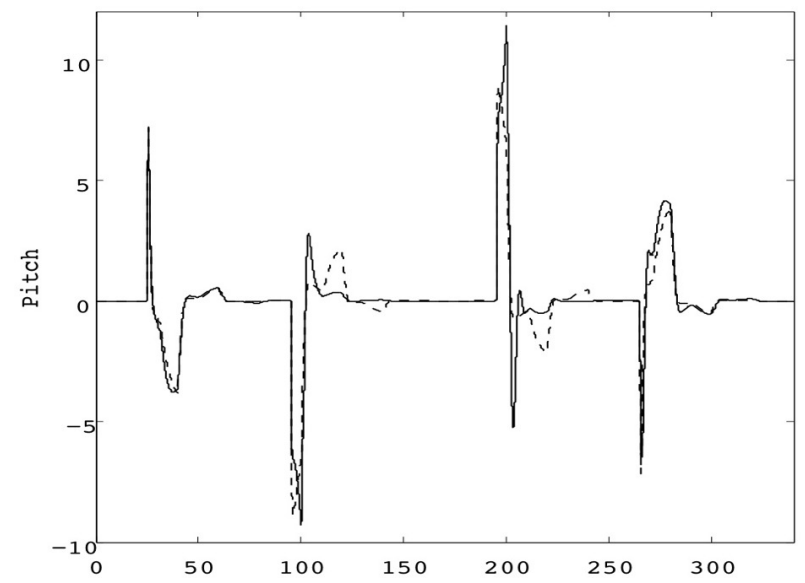

Figure 3: Times histories of pitch angle $\theta(t)$

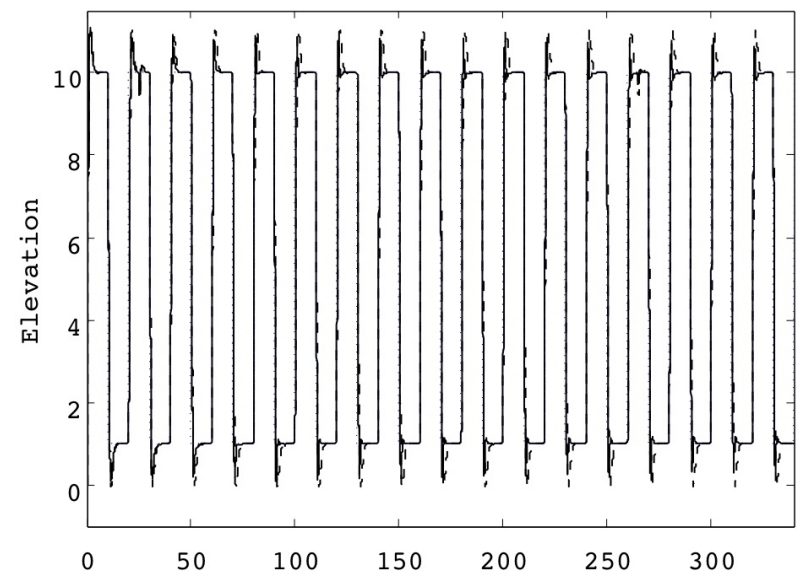

Figure 4: Times histories of elevation angle $\epsilon(t)$ 


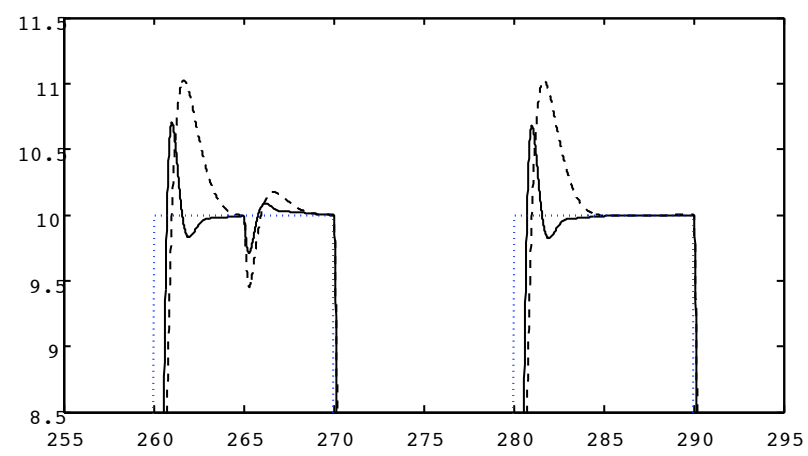

Figure 5: Zoom on times histories of elevation angle $\epsilon(t)$

Comparisons of the static PID and the adaptive control law do not give any decisive conclusion on the superiority of one or the other. Experiments on the real plant will be done by the time of the final submission of the paper and may give some more insight on the respective properties. We expect to recover properties we had reported in a former paper ([AFP07]). Meanwhile, experiments have been conducted including noise on the measurements. No major difference is noted compared to the given figures.
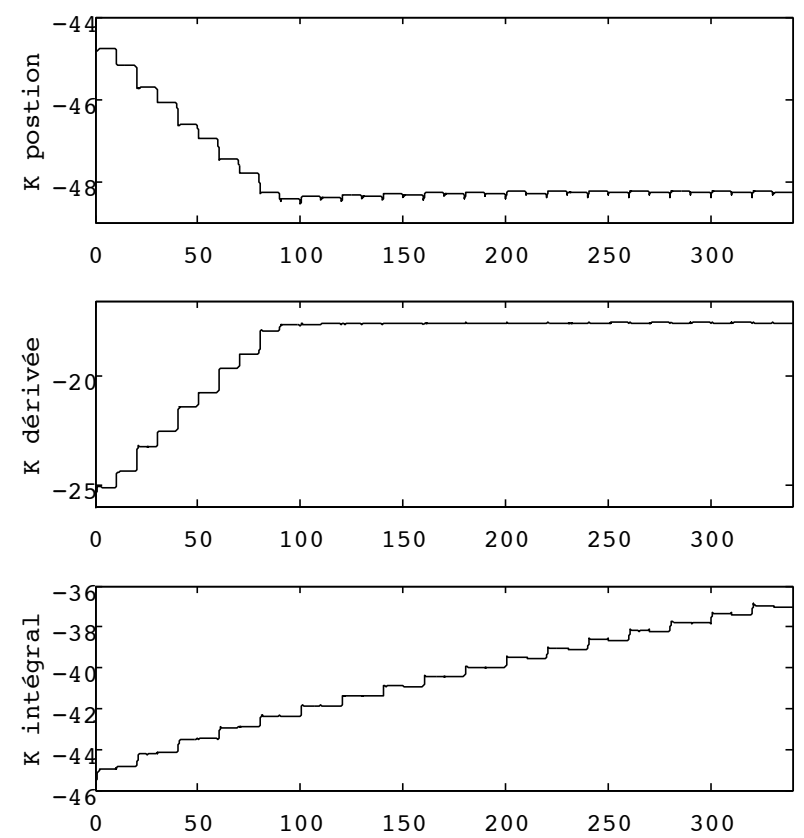

Figure 6: Times histories of adaptive PID $K_{v}(t)$ for elevation angle

Figures 6 and 7 give the time histories of the gains $K_{v}$ (adaptive PID for the elevation axis) and $K_{u}$ (adaptive PID for the travel-pitch angles). It shows that the domains in which the gains are bounded do allow quite large variations. When the limits of this region are reached (as in Figure 6) the barrier functions do constrain the gains to keep in the region. 


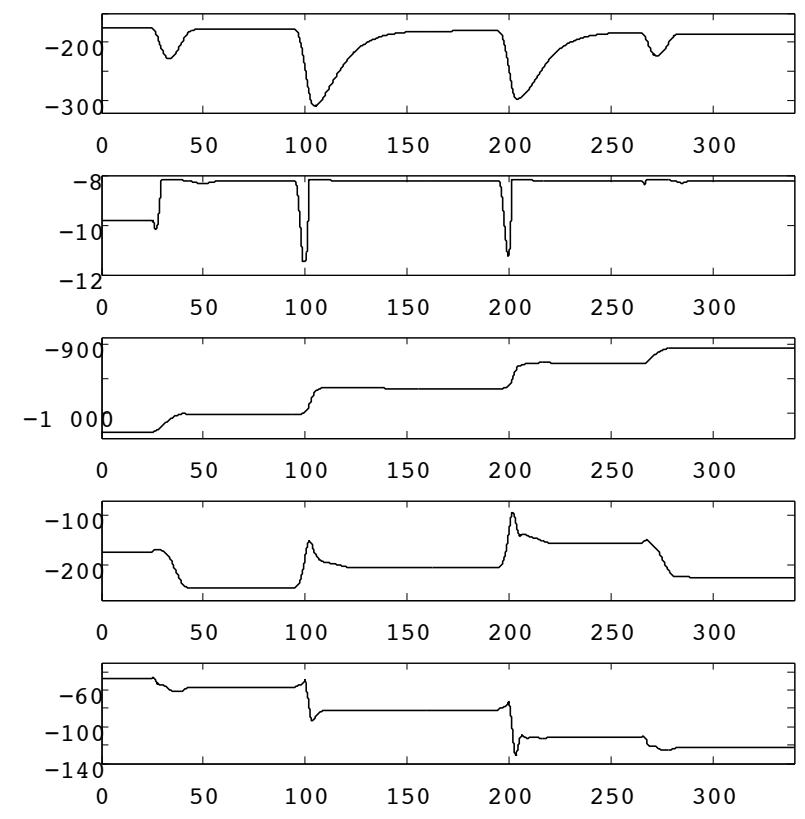

Figure 7: Times histories of adaptive PID $K_{u}(t)$ for travel-pitch angles

\section{Conclusion}

A new theoretical result is provided for designing robust adaptive controllers. The method only needs the knowledge of a stabilizing static gain thus relaxing passivity assumptions. Dividing the adaptive lax in two parts involving different signals the method also allows to build adaptive PID controllers. The methodology is satisfactorily applied on the model of a helicopter benchmark. It validates the design procedure. The advantages of the adaptive control are not straightforward on the simulations. Experiments on the benchmark that will be done before the final submission of the paper may give more insight with respect to this comparison.

\section{References}

[AFP07] B.R. Andrievsky, A.L. Fradkov, and D. Peaucelle. Adaptive control of 3DOF motion for LAAS helicopter benchmark: design and experiments. In American Control Conference, New-York, July 2007.

[ÅW89] K.J. Åström and B. Wittenmark. Adaptive Control. Addison-Wesley, 1989.

[BTH06] I. Barkana, M.C.M. Teixeira, and L. Hsu. Mitigation of symmetry condition in positive realness for adaptive control. Automatica, 42(9):1611-1616, September 2006.

[Fra74] A.L. Fradkov. Adaptive stabilization of a linear dynamic plant. Autom. Remote Contr., 35(12):19601966, 1974.

[Fra03] A.L. Fradkov. Passification of non-square linear systems and feedback Yakubovich-Kalman-Popov lemma. European J. of Control, 6:573-582, 2003.

[Fra08] A.L. Fradkov. Passification of linear systems with respect to given output. In IEEE Conf. Decision and Control, pages 646-651, 2008.

[IS96] P. Ioannou and J. Sun. Robust Adaptive Control. Prentice Hall, Inc, 1996.

[KBS94] H. Kaufman, I. Barkana, and K. Sobel. Direct adaptive control algorithms. Springer, New York, 1994. 
[KKGZ06] T. Kiefer, A. Kugi, K. Graichen, and M. Zeitz. Feedforward and feedback tracking control of a 3 DOF helicopter experiment under input and state/output constraints. In IEEE Conf. Decision and Control, pages 1586-1593, San Diego, décembre 2006.

[KKK04] T. Kiefer, A. Kugi, and W. Kemmetmüller. Modeling and flatness-based control of 3DOF helicopter laboratory experiment. In IFAC Symposium on Nonlinear Control Systems, pages 207-212, Stuttgart, septembre 2004.

[Löf04] J. Löfberg. YALMIP : A Toolbox for Modeling and Optimization in MATLAB, 2004.

[PA06] D. Peaucelle and D. Arzelier. Robust multi-objective control toolbox. In IEEE International Symposium on Computer-Aided Control Systems Design, Munich, October 2006.

[PDPM11] D. Peaucelle, A. Drouot, C. Pittet, and J. Mignot. Simple adaptive control without passivity assumptions and experiments on satellite attitude control DEMETER benchmark. In IFAC World Congress, August 2011.

[Pea05] D. Peaucelle. RoMulOC: a YALMIP-Matlab based robust multi-objective control toolbox. Technical Report 05377, LAAS-CNRS, Toulouse, June 2005.

[PF08] D. Peaucelle and A.L. Fradkov. Robust adaptive $L_{2}$-gain control of polytopic MIMO LTI systems LMI results. Systems \& Control Letters, 57(11):881-887, 2008.

[PFA07] D. Peaucelle, A. Fradkov, and B. Andrievsky. Adaptive identification of angular motion model parameters for LAAS helicopter benchmark. In IEEE Conference on Control Application, Singapore, October 2007.

[PFA08] D. Peaucelle, A. Fradkov, and B. Andrievsky. Passification-based adaptive control of linear systems: Robustness issues. Int. J. of Adaptive Control and Signal Processing, 22(6):590-608, August 2008. doi: 10.1002/acs.1009.

[PKP09] D. Peaucelle, H.M. Khan, and P.V. Pakshin. LMI-based analysis of robust adaptive control for linear systems with time-varying uncertainty. Autom. Remote Contr., 70(9):1540-1552, 2009.

[Stu99] J.F. Sturm. Using SeDuMi 1.02, a MATLAB toolbox for optimization over symmetric cones. Optimization Methods and Software, 11-12:625-653, 1999. 